Pacific Journal of Mathematics






\title{
TWO APPLICATIONS OF THE SCHUR-NEVANLINNA
}

\section{ALGORITHM}

\author{
ARNE STRAY
}

\begin{abstract}
Two applications of the Schur-Nevanlinna algorithm are given. The first application gives new information about the Nevanlinna-Pick interpolation problem. The second application concerns the constructive approximation to bounded measurable functions on the unit circle by functions from $H^{\infty}$.
\end{abstract}

1. Introduction. The Schur-Nevanlinna algorithm was developed by I. Schur [7] and refined by R. Nevanilnna [6] in the study of certain interpolation problems for bounded analytic functions.

The main idea in the first application is to combine Nevanlinna's algorithm with a certain uniqueness criterion due to Denjoy. This gives new information about solutions of the Nevanlinna-Pick interpolation problem.

The second application concerns the constructive approximation to bounded measurable functions on the unit circle $T=\{z:|z| \leqq 1\}$, by functions from $H^{\infty}$. As usual, $H^{\infty}$ consists of the bounded analytic functions in the unit disc $D=\{z:|z|<1\}$. They are extended to $D \cup T$ by taking radial limits, thanks to a well known theorem of Fatou. The main tool here is Schur's algorithm [7]. Assuming the recent result about duality between $H^{1}$ and BMO [1], our method also yields a constructive decomposition of functions $f$ in the class BMO (functions of bounded mean oscillation). This has recently been done by $\mathrm{P}$. Jones [4], using entirely different methods.

2. Nevanlinna's algorithm and Denjoy's criterion. It will be necessary to describe the results and ideas in Nevanlinna's fundamental paper [6], in some detail.

The space $H^{\infty}$ introduced above, is a Banach space with the norm $\|f\|=\sup \{|f(z)|, z \in D\}$. Let $\left\{z_{\nu}\right\}$ be a sequence of distinct points in $D$, and consider the interpolation problem

$$
w\left(z_{\nu}\right)=w_{\nu}, \quad \nu=1,2, \cdots
$$

where $\left\{w_{\nu}\right\}$ is a specified sequence of complex numbers, and $w \in H^{\infty}$, $\|w\| \leqq 1$.

We assume that $\left({ }^{*}\right)$ has at least two solutions. Then $R$. Nevanlinna [6] has shown that all solutions to $\left({ }^{*}\right)$ are given by the following formula 
$(* *)$

$$
w=\frac{P-Q w_{\infty}}{R-S w_{\infty}}
$$

where $w_{\infty}$ is an arbitrary function in the unit ball of $H^{\infty}$, and $P$, $Q, R$ and $S$ are certain analytic functions in $D$. If we only consider interpolation at the $N$ first points $z_{1}, \cdots, z_{N}$, the corresponding functions in (**) will be denoted by $P_{N}, Q_{N}, R_{N}$ and $S_{N}$ respectively.

Nevanlinna used a normal family argument to show that $P_{N}$, $Q_{N}, R_{N}$ and $S_{N}$ converge uniformly on compact subsets of $D$, to their respective units $P, Q, R$ and $S$. Recently M. Heins [2] has treated such convergence questions in a more general situation.

Using Denjoy's criterion, we deduce stronger convergence properties on $P_{N}, Q_{N}, R_{N}$ and $S_{N}$ than previously known.

Let $W=C \cup\{\infty\} \backslash E$, where $E$ is the closure of the set $\left(\bar{z}_{\nu}{ }^{-1}\right.$, $\nu=1,2, \cdots\}$. We assume that $T \backslash E$ is nonempty.

THEOREM. The functions $P, Q, R$ and $S$ extends to be analytic in $W$, and the convergence of $P_{N}, Q_{N}, R_{N}$ and $S_{N}$, to $P, Q, R$ and $S$ respectively, is uniform on compact subsets of $W$.

To prove this theorem, we have to combine various formulas from Nevanlinna's paper [6]. Some of these formulas will also be useful in $\S 3$.

Nevanlinna gives a representation of all functions $w$ in the unit ball of $H^{\infty}$, such that

$$
w\left(z_{\mu}\right)=w_{\mu}, \quad 1 \leqq \mu \leqq \nu
$$

The formula is

$$
w=\frac{A_{\nu}-B_{\nu} w_{\infty}}{C_{\nu}-D_{\nu} w_{\infty}}
$$

where $A_{\nu}, B_{\nu}, C_{\nu}$ and $D_{\nu}$ are polynomials of degree not greater than $\nu$, and $w_{\infty}$ is an arbitrary function in the unit ball of $H^{\infty}$.

These polynomials are obtained from a recursive set of equations which will be important to us. Before describing these equations, we remark that $A_{\nu}, B_{\nu}, C_{\nu}$ and $D_{\nu}$ differ from $P_{\nu}, Q_{\nu}, R_{\nu}$ and $S_{\nu}$ by a common rational factor $\lambda_{\nu}$ which we define below. If we put $A_{0}=$ $D_{0}=0, B_{0}=1$, and $C_{0}=-1$, the recursive equations can be written in matrix form

$$
\left[\begin{array}{ll}
A_{\nu} & B_{\nu} \\
C_{\nu} & D_{\nu}
\end{array}\right]=\left[\begin{array}{ll}
A_{\nu-1} & B_{\nu-1} \\
C_{\nu-1} & D_{\nu-1}
\end{array}\right] M_{\nu}, \quad \nu=1,2, \cdots
$$

where $M_{\nu}$ is a $2 \times 2$ matrix depending more or less on the original 
interpolation data for the problem (*). In fact we have

$$
M_{\nu}=\left[\begin{array}{ll}
\left(s_{\nu}-r_{\nu} z \varepsilon_{\nu}\right) & \left(q_{\nu}+p_{\nu} z \varepsilon_{\nu}\right) \eta_{\nu} \\
-\left(p_{\nu}+q_{\nu} z \varepsilon_{\nu}\right) \bar{\eta}_{\nu} & r_{\nu}-s_{\nu} z \varepsilon_{\nu}
\end{array}\right]
$$

where

$$
\begin{aligned}
& p_{\nu}=b_{\nu}-a_{\nu} c_{\nu} \\
& q_{\nu}=c_{\nu}-a_{\nu} b_{\nu} \\
& r_{\nu}=a_{\nu}-b_{\nu} c_{\nu} \\
& s_{\nu}=1-a_{\nu} b_{\nu} c_{\nu} .
\end{aligned}
$$

The numbers $a_{\nu}, b_{\nu}$ and $c_{\nu}$ belong to the interval $[0,1]$ and we have

$$
\begin{aligned}
z_{\nu} & =a_{\nu} \bar{亏}_{\nu}=a_{\nu} e^{i \alpha_{\nu}} \\
w_{\nu}^{(\nu)} & =b_{\nu} \bar{\eta}_{\nu}=b_{\nu} e^{i \beta_{\nu}}
\end{aligned}
$$

where $w_{\nu}^{(\nu)}$ is a certain well defined rational function of the interpolation data $w_{1}, \cdots, w_{\nu}\left([6]\right.$, p. 37). The numbers $\left\{c_{\nu}\right\}$ can be chosen freely from $[0,1]$ but in the following we assume $c_{\nu}=a_{\nu} b_{\nu}, \nu=$ $1,2, \cdots$. This is the so called Denjoy normalization ([6], page 39) which has many advantages.

The rational functions $P_{\nu}, Q_{\nu}, R_{\nu}$ and $S_{\nu}$ are now defined by

$$
\left[\begin{array}{ll}
P_{\nu} & Q_{\nu} \\
R_{\nu} & S_{\nu}
\end{array}\right]=\lambda_{\nu}\left[\begin{array}{ll}
A_{\nu} & B_{\nu} \\
C_{\nu} & D_{\nu}
\end{array}\right]
$$

where $\lambda_{\nu}(z)=\prod_{\mu=1}^{\nu}\left(\left(1-b_{\mu}^{2}\right)\left(1-c_{\mu}^{2}\right)\right)^{-1 / 2}\left(1-\bar{z}_{\mu} z\right)^{-1}$.

These rational functions are more convenient to work with than $A_{\nu}, B_{\nu}, C_{\nu}$ and $D_{\nu}$ for several reasons.

For example, it is not hard to show that $A_{\nu} D_{\nu}-B_{\nu} C_{\nu}$ vanishes at $z_{1}, \cdots, z_{\nu}$ ([6], page 32), but the factor $\lambda_{\nu}$ has the effect that

$$
P_{\nu} S_{\nu}-R_{\nu} Q_{\nu}=\pi_{\nu}
$$

where $\pi_{\nu}=\prod_{\mu=1}^{\nu}\left(a_{\mu}-z \varepsilon_{\mu}\right) /\left(1-a_{\mu} z \varepsilon_{\mu}\right)$ is the Blaschke product corresponding to $\left\{z_{\mu}\right\}_{\mu=1}^{\nu}$. The Blaschke product corresponding to $\left\{z_{\nu}\right\}_{\nu=1}^{\infty}$ is denoted by $\pi$.

We need the following additional equations from [6], (see pages 36 and 43):

$$
\begin{aligned}
& A_{\nu}(z)=(-1)^{\nu+1} e^{i \theta_{\nu}} z_{\nu}^{\nu} \overline{D_{\nu}\left(\frac{1}{\bar{z}}\right)} \\
& B_{\nu}(z)=(-1)^{\nu+1} e^{i \theta_{\nu}} z^{\nu} C_{\nu}\left(\frac{1}{\bar{z}}\right)
\end{aligned}
$$

(5) $\left|R_{\nu}\right| \geqq 1$ and $\left.\max \left\{\left|P_{\nu}(z)\right|,\left|Q_{\nu}(z)\right|,\left|S_{\nu}(z)\right|\right\} \leqq\left|R_{\nu}(z)\right|\right\}, \quad z \in D$. 
REMARK. In (4), $\theta_{\nu}=-\sum_{\mu=1}^{\nu} \alpha_{\mu}=-\sum_{\mu=1}^{\nu} \arg z_{\mu}$. The factor $(-1)^{\nu}$ seems to be missing in the corresponding formula in [6]. Both (4) and (5) reflect the fact that for fixed $z \in D$, the transformation

$$
w \longrightarrow \frac{P_{\nu}(z)-Q_{\nu}(z) w}{R_{\nu}(z)-S_{\nu}(z) w}
$$

carries $D$ into $D$, and that the mapping is onto if $|z|=1$.

We can now give the proof of Theorem 1 .

If we combine equations (3) and (4), it is easy to verify that

$$
\begin{aligned}
& P_{\nu}(z)=-\pi_{\nu}(z) \overline{S_{\nu}\left(\frac{1}{\bar{z}}\right)} \\
& Q_{\nu}(z)=-\pi_{\nu}(z) \overline{R_{\nu}\left(\frac{1}{\bar{z}}\right)} .
\end{aligned}
$$

If we express the recursive equation (2) in terms of $P_{\nu}, Q_{\nu}, R_{\nu}, S_{\nu}$, we can write it in the form

$$
\left[\begin{array}{ll}
P_{\nu} & Q_{\nu} \\
R_{\nu} & S_{\nu}
\end{array}\right]=\left[\begin{array}{ll}
P_{\nu-1} & Q_{\nu-1} \\
R_{\nu-1} & S_{\nu-1}
\end{array}\right]\left(I+\varepsilon_{\nu}\right)
$$

where $I$ is the identity matrix, and $\varepsilon_{\nu}$ is a matrix whose size is easy to estimate thanks to the following result due to Denjoy ([6], p. 42).

Denjoy's criterion. The problem (*)has more than one solution if and only if $\sum_{\nu=1}^{\infty}\left(1-a_{\nu}\right)\left(1-b_{\nu}\right)^{-1}<\infty$.

Instead of estimating the norm of $\varepsilon_{\nu}$, let us be content to consider the left lower corners in (7) only. We then get

$$
R_{\nu}=\left(1+t_{\nu}\right) R_{\nu-1}+t_{\nu}^{\prime} S_{\nu-1}
$$

where

$$
1+t_{\nu}(z)=\frac{\left(1-b_{\nu}^{2}\right)\left(1-\bar{z}_{\nu} z\right)+b_{\nu}^{2}\left(1-a_{\nu}^{2}\right)}{\left(1-b_{\nu}^{2}\right)^{1 / 2}\left(1-a_{\nu}^{2} b_{\nu}^{2}\right)^{1 / 2}\left(1-\bar{z}_{\nu} z\right)}
$$

and

$$
t_{\nu}^{\prime}=\frac{b_{\nu}\left(1-a_{\nu}^{2}\right)}{\left(1-b_{\nu}^{2}\right)^{1 / 2}\left(1-a_{\nu}^{2} b_{\nu}^{2}\right)^{1 / 2}\left(1-\bar{z}_{\nu} z\right)} .
$$

Since $\left(1-a_{\nu}^{2} b_{\nu}^{2}\right) /\left(1-b_{\nu}^{2}\right)=1+b_{\nu}^{2}\left(1-a_{\nu}^{2}\right) /\left(1-b_{\nu}^{2}\right)$, it follows that the inequality

$$
\left|t_{\nu}(z)\right|+\left|t_{\nu}^{\prime}(z)\right| \leqq C_{K} \frac{1-a_{\nu}}{1-b_{\nu}}
$$


holds uniformly on any compact subset $K$ of $W$, with $C_{K}$ depending on $K$, but not on $\nu$.

From (8) we have

$$
1=\left(1+t_{\nu}\right) \frac{R_{\nu-1}}{R_{\nu}}+t_{\nu}^{\prime} \frac{S_{\nu-1}}{R_{\nu-1}} \frac{R_{\nu-1}}{R_{\nu}} .
$$

In light of (5), we conclude that

$$
\frac{R_{\nu}}{R_{\nu-1}}(z)=1+0\left(\frac{1-a_{\nu}}{1-b_{\nu}}\right)
$$

holds uniformly in $K \cap\{z:|z| \leqq 1\}$. By Denjoy's eriterion $R_{\nu} \rightarrow \Omega$ uniformly on compact subsets of $W \cap\{z:|z| \leqq 1\}$.

Let us assume that $K$ has the symmetry property $z \in K=\bar{z}^{-1} \in K$. If $z \in K \cap\{z:|z| \leqq 1\}$, (5) and (6) gives

$$
\left.R_{\nu}\left(\bar{z}^{-1}\right)|\leqq| \pi_{\nu}(z)\right|^{-1}\left|Q_{\nu}(z)\right| \leqq\left|\pi_{\nu}(z)\right|^{-1}\left|R_{\nu}(z)\right| \leqq C_{\Lambda}^{\prime},
$$

where $C_{K}^{\prime}$ depends only on $K$ and $R$, since $\pi_{\nu} R_{\nu} \rightarrow \pi R$ uniformly on $K \cap\{z:|z| \leqq 1\}$. Since $W$ is connected, the last inequality implies that $\left\{R_{\nu}\right\}$ converges uniformly on compact subsets of $W$.

The convergence of $\left\{Q_{2}\right\}$ follows easily from (6). From (T) we also have the identity

$$
P_{2}=\left(1+t_{2}\right) P_{2-1}+t_{2}^{\prime} Q_{\nu-1}
$$

which implies (in light of (5)) that

$$
\left|P_{\nu}-P_{\nu-1}\right| \leqq\left|t_{\nu}\right|\left|P_{\nu-1}\right|+\left|t_{\nu}^{\prime}\right|\left|Q_{-1}\right| \leqq\left|R_{\nu}\right|\left(\left|t_{\nu}\right|+\left|t_{\nu}^{\prime}\right|\right) \text {. }
$$

Using what we just have proved about $\left\{R_{2}\right\}$, we see that $\sum\left(P_{\nu}-P_{2-1}\right)$ converges uniformly on compact subsets on $W \cap$ $\{z:|z| \leqq 1\}$. The convergence of $\left\{P_{*}\right\}$ and $\left\{S_{\psi}\right\}$ in $W$ now follows easily.

We recall that an inner function in $H^{\infty}$ is characterized by having unimodular radial limits almost everywhere on $T$.

Corollary. Assume there are at least two solutions to the interpolation problem (*) with minimal norm. Then there is a minimal solution which is a constant times an inner function analytic across $T / E$.

Proof. We may assume that the minimal norm is one. If we choose $w_{\infty} \equiv \zeta$ in (**), where $\zeta \in T$ is a constant, Nevanlinna ([6], p. 48) showed that $w$ given by (**), is an inner function. The analyticity across $T \backslash E$ follows from the above theorem. The above corollary was apparently proved first by D. Hejal [3], and he even 
proved such a result for finitely connected domains with smooth boundary.

Let us finally remark that if the assumption about non-uniqueness is dropped in the corollary, no conclusion can be drawn. In fact, it was recently proved by $\mathrm{K}$. 0 . Øyma and the present author (and independently by D. Marshall) that any extreme point in the unit ball of $H^{\infty}$, is the unique minimal solution to an interpolation problem $\left({ }^{*}\right)$, where $\left\{z_{\nu}\right\}$ has only one limit point on $T$. (See [8].)

Clearly such a function $h$ may not satisfy any of the conclusions in the above corollary. On the other hand, if $h$ is not extreme, it can never be a unique minimal solution to a problem like $\left(^{*}\right)$.

3. Schur's algorithm, and a problem in constructive approximation. Consider a polynomial

$$
p_{N}(z)=C_{1} z+C_{2} z^{2}+\cdots+C_{N} z^{N}
$$

and associated with it, the set

$$
E_{N}=\left\{h \in H^{\infty}:\left\|p_{N}-z^{N+1} h\right\| \leqq 1\right\} .
$$

Let $S_{N}=\left\{p_{N}-z^{N+1} h: h \in E_{N}\right\}$. Then Schur [7] showed that if $E_{N} \neq \phi$, then $S_{N}$ could be parametrized by the formula (1) given above:

$$
p_{N}-z^{N+1} h=\frac{A_{N}-B_{N} w_{\infty}}{C_{N}-D_{N} w_{\infty}} .
$$

REMARK. Actually Schur's formula looks a little bit different from the above one, since his notation is slightly different from Nevanlinna's. We shall not write out Schur's formulas in detail, but be content to mention the following.

Schur's algorithm (a simpler, limiting case of the recursive equations (2) mentioned above) provides the following, after a finite number of computations involving the coefficients $C_{1}, \cdots, C_{N}$ of $p_{N}$ :

(i) It shows whether the set $E_{N}$ is empty or not;

(ii) It gives an explicit construction of the polynomials $A_{N}$, $B_{N}, C_{N}$ and $D_{N}$ in (10), and in particular we mention: $C_{N}(0)>0$, $D_{N}(0)=0$, and $A_{N} D_{N}-B_{N} C_{N}=\gamma_{N} z^{N+1}$, where $\gamma_{N}$ is a positive constant computed in terms of the coefficients of $p_{N}$.

Now let $F \in L^{\infty}$. We assume $F \notin H^{\infty}$, and shall construct $H \in H^{\infty}$, such that

$$
\|F-H\|_{\infty} \leqq 4 d\left(F, H^{\infty}\right)
$$

where $d\left(F, H^{\infty}\right)=\inf \left\{\|F-h\|_{\infty}, h \in H^{\infty}\right\}$. The norm considered here, is the usual essential supremum norm on $L^{\infty}(T)$. We recall that $H^{\infty}$ 
can be viewed as a $w^{*}$-closed subalgebra of $L^{\infty}(T)$, and that the sup norm on $D$ coincides with $\|h\|_{\infty}$ if $h \in H^{\infty}$.

Let $F$ be expanded in its Fourier series

$$
F\left(e^{i \theta}\right) \sum_{k=-\infty}^{\infty} a_{k} e^{i k \theta}
$$

and form $F_{N}=F * \sigma_{N}$, where $\sigma_{N}$ denotes the $N$ th Fejer kernel.

Then

$$
F_{N}\left(e^{i \theta}\right)=\sum_{k=-N}^{N}\left(1-\frac{|k|}{N+1}\right) a_{k} e^{i k \theta} .
$$

Now let $p_{N}$ be the polynomial related to $F_{N}$ by putting

$$
p_{N}\left(e^{i \theta}\right)=e^{i(N+1) \theta} \sum_{k=-N}^{-1}\left(1-\frac{|k|}{N+1}\right) a_{k} e^{i k \theta} .
$$

Then clearly

$$
d\left(F_{N}, H^{\infty}\right)=\inf \left\{\left\|p_{N}-z^{N+1} h\right\|_{\infty}, h \in H^{\infty}\right\} .
$$

If we apply Schur's algorithm to $2^{n} p_{N}$, where $n$ runs through the integers, we can determine a unique integer $k_{N}$ such that

$$
2^{k_{N}}<d\left(F_{N}, H^{\infty}\right) \leqq 2^{k_{N+1}} .
$$

Note that since $d\left(F_{N}, H^{\infty}\right) \rightarrow d\left(F, H^{\infty}\right)$ as $N \rightarrow \infty$, there will exist an integer $k$ such that $k_{N}=k$ eventually, and such that (11) holds with $k_{N}$ replaced by $k$ and $F_{N}$ by $F$.

Let us put $\widetilde{F}_{N}=2^{-k_{N}-2} F_{N}$, such that $2^{-2} \leqq d\left(\widetilde{F}_{N}, H^{\infty}\right) \leqq 2^{-1}$, and apply the Schur algorithm to $\widetilde{p}_{N}=2^{-k_{N}-2} p_{N}$.

The set $\left\{h \in H^{\infty}:\left\|\widetilde{p}_{N}-Z^{N+1} h\right\|_{\infty} \leqq 1\right\}$, will still be denoted by $E_{N}$.

Formula (10) above, and the remarks (i) and (ii) about Schur's algorithm, applies to $\widetilde{p}_{N}$, and we have for $h \in E_{N}$ :

$$
\tilde{p}_{N}-z^{N+1} h=\frac{A_{N}}{C_{N}}-\frac{\left(A_{N} D_{N}-B_{N}(N) w_{\infty}\right.}{C_{N}\left(C_{N}-D_{N} w_{\infty}\right)}=\frac{A_{N}}{C_{N}}-\frac{\gamma_{N} z^{N+1} B_{N} w_{\infty}}{C_{N}\left(C_{N}-D_{N} w_{\infty}\right)} .
$$

If we consider $\max \left\{\operatorname{Re} h(0), h \in E_{N}\right\}$, the last expression shows that this maximum is attained by a unique function $h_{N}$ corresponding to $w_{\infty} \equiv-1$. (Note that $C_{N}(0)>0$ and $D_{N}(0)=0$.)

Define now $\widetilde{H}_{N} \in H^{\infty}$ by the relation

$$
\widetilde{F}_{N}-\widetilde{H}_{N}=\frac{1}{z^{N+1}} \widetilde{p}_{N}-h_{N}
$$

Then $\left\|\widetilde{F}_{N}-\widetilde{H}_{N}\right\|_{\infty} \leqq 1$, and $\operatorname{Re} H_{N}$ is maximal among all $h \in H^{\infty}$ such that $\left\|F_{N}-h\right\|_{\infty} \leqq 1$. 
Let $\widetilde{H}$ be any $w^{*}$ cluster point of $\widetilde{H}_{N}$. Then clearly $\|\widetilde{F}-\widetilde{H}\|_{\infty} \leqq 1$. We claim that $\operatorname{Re} \tilde{H}(0)$ is maximal among all $h \in H^{\infty}$ such that $\|\widetilde{F}-h\|_{\infty} \leqq 1$. This follows since $\widetilde{H}_{N}(0) \rightarrow \widetilde{H}(0)$, and from our choice of $\tilde{H}_{N}$.

But then $|\widetilde{F}-\widetilde{H}|=1$ almost everywhere on $T$ as Garnett has shown [5]. This implies that $\widetilde{H}$ is unique, since a convex combination of two different unimodular functions fails to be unimodular.

So the sequence $\left\{H_{N}\right\}$, given by $H_{N}=2^{k_{N}+2} \widetilde{H}_{N}$, converges uniformly on compact subsets of $\{z:|z|<1\}$ to a function $H \in H^{\infty}$, such that $\|F-H\|_{\infty} \leqq 4 d\left(F, H^{\infty}\right)$.

Final remarks, Recently P. Jones [4] gave a constructive proof of the fact that any functions $f$ in the class BMO (Functions of bounded mean oscillation) can be decomposed as

$$
f=u+\tilde{v}
$$

where $u, v \in L^{\infty}$, and $\widetilde{v}$ denotes the harmonic conjugate of $v$.

This decomposition appeared first in the fundamental paper [1] ba C. Fefferman and E. Stein, but no constructive proof was known. Jones' proof is constructive and geometrical in nature, and combines the theory of interpolation by bounded analytic functions with recent work by N. Th. Varopoulos [9], [10].

As Jones points out [4], this problem is much related to the broblem of estimating the distance a functions $F \in L^{\infty}$, to $H^{\infty}$.

We want to indicate briefly how the decomposition $f=u+\widetilde{v}$ can be obtained constructively using our approach above. For basic properties and definition of BMO, see [1] and [4].

Using either the results of Varopolous [9], [10] or FeffermanStein's result that BMO can be realized as the dual of the Hardy space $H^{1}$, we have the inequality

$$
d\left(F, H^{\infty}\right) \leqq C\|F\|_{*}
$$

where $C$ is an absolute constant and " \|\|$_{*}$ " denotes the BMO-norm.

So let $f \in$ BMO be real valued, with $\|f\|_{*} \leqq 1$. If $\sigma_{N}$ is the $N^{\prime}$ th Fejer kernel, let $f_{N}=f * \sigma_{N}$. Since the norm " \|\|$_{*}$ " is rotation invariant

$$
\left\|f_{N}\right\|_{*} \leqq\|f\|_{*} \leqq 1
$$


and hence

$$
d\left(f_{N}, H^{\infty}\right) \leqq\left\|f_{N}\right\|_{*} \leqq C
$$

for all $N$.

We now construct $h_{N} \in H^{\infty}$ with $\left\|f_{N}-h_{N}\right\|_{\infty} \leqq 4 d\left(f_{N}, H^{\infty}\right) \leqq 4 C$, and with $\operatorname{Re} h_{N}(0)$ maximal among all $h \in H^{\infty}$ satisfying the last inequality.

We now write

$$
f_{N}=\left(f_{N}-h_{N}\right)+h_{N} .
$$

Since $\operatorname{Im} h_{N}$ is bounded, we can use a compactness argument and obtain that $\left\{h_{N}\right\}$ has a subsequence converging uniformly on compact subsets of $\{|z|<1\}$ to a function $h$ analytic in $D$, such that

$$
\|f-h\|_{\infty} \leqq 4 C \text {. }
$$

This does not seem very constructive, but we are saved by the fact that $\operatorname{Re} h(0)$ is maximal among all holomorphic functions $b$ in $\{|z|<1\}$ satisfying $\|f-b\| \leqq 4 C$, and this specifies $h$ uniquely as it did in $\S 3$ where we dealt with a bounded function $F$ instead of $f$.

So $h_{N} \rightarrow h$ uniformly on compact subsets of $D$, and if we take real part in the decomposition

$$
f=f-h+h,
$$

we have

$$
f=\operatorname{Re}(f-h)+(\widetilde{\operatorname{Im} h}),
$$

which gives the desired decomposition.

Our decomposition is constructive, but not geometrical. On the other hand, there are BMO-functions which can be given rather explicitely in terms of certain lacunary Fourier series, and for such functions, the above construction may be of some use.

\section{REFERENCES}

0. P. Duren, Theory of $H^{p}$ Spaces, Academic Press, 1971.

1. C. Fefferman and E. M. Stein, $H^{p}$ spaces of several variables, Acta Math., 129 (1972), 138-193.

2. M. Heins, On W.S.N. functions and a theorem of $R$. Nevanlinna concerning bounded analytic functions, Kodai Math. Sem. Rep., 26 (1975), 245-257.

3. D. Hejal, Linear extremal problems for analytic functions with inside conditions, Ann. Acad. Sci. Fenn., Ser. A 586 (1975), 1-36.

4. P. Jones, $A$ constructive decomposition of $\mathrm{BMO}(R)$ into $L^{\infty}$ plus Hilbert transform of $L^{\infty}$, preprint.

5. D. Marshall, Thesis, U.C. L.A., page 12.

6. R. Nevanlinna, Über beschränkte analytische Funktionen, Ann. Acad. Sci. Fenn., Ser. A 32 (1929), 1-7. 
7. I. Schur, Journal fur Math. 148 (1918), 122-145.

8. A. Stray and K. O. Oyma, On interpolating functions of minimal norm, Proc. Amer Math. Soc., 68 (1978), 75-78.

9. N. Th. Varopoulos, BMO functions and the $\bar{\sigma}$ equation, Pacific J. Math. 71 (1977), 221-273.

10. - A remark on BMO and bounded harmonic functions, Pacific J. Math., 73 (1977), 257-259.

Received January 30, 1979 and in revised from September 24, 1979.

UNIVERSITY OF MARYLAND

College Park, MD 20742 


\section{PACIFIC JOURNAL OF MATHEMATICS}

\section{EDITORS}

DoNALD BABBITT (Managing Editor)

University of Galifornia

Los Angeles, California 90024

HUgo RossI

University of Utah

Salt Lake City, UT 84112

C. C. MOORE AND ANDREW OGG

University of California

Berkeley, CA 94720
J. DugunduI

Department of Mathematics

University of Southern California

Los Angeles, California 90007

R. Finn and J. Milgram

Stanford University

Stanford, California 94305

\section{ASSOCIATE EDITORS}
E. F. BeCKenbaCh
B. H. NeumanN
F. WOLF
K. YoSHIDA

\section{SUPPORTING INSTITUTIONS}

UNIVERSITY OF BRITISH COLUMBIA

CALIFORNIA INSTITUTE OF TECHNOLOGY

UNIVERSITY OF CALIFORNIA

MONTANA STATE UNIVERSITY

UNIVERSITY OF NEVADA, RENO

NEW MEXICO STATE UNIVERSITY

OREGON STATE UNIVERSITY

UNIVERSITY OF OREGON
UNIVERSITY OF SOUTHERN CALIFONIA STANFORD UNIVERSITY UNIVERSITY OF HAWAII UNIVERSITY OF TOKYO UNIVERSITY OF UTAH WASHINGTON STATE UNIVERSITY UNIVERSITY OF WASHINGTON 


\section{Pacific Journal of Mathematics}

\section{Vol. 91, No. $1 \quad$ November, 1980}

Harvey Leslie Abbott, Extremal problems on nonaveraging and nondividing

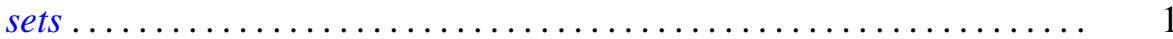

Marine Bruce Abrahamse and Stephen D. Fisher, Mapping intervals to

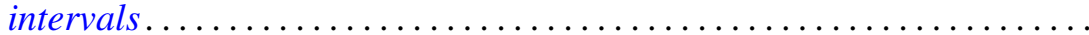

William Wells Adams, The best two-dimensional Diophantine

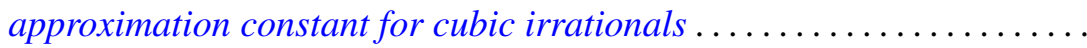

Marilyn Breen, A quantitative version of Krasnosel'skiu 's theorem in

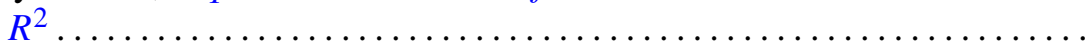

Stephen LaVern Campbell, Linear operators for which $T^{*} T$ and $T T^{*}$

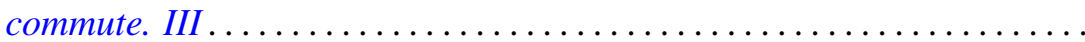

Zvonko Cerin, On cellular decompositions of Hilbert cube manifolds ......

J. R. Choike, Ignacy I. Kotlarski and V. M. Smith, On a characterization

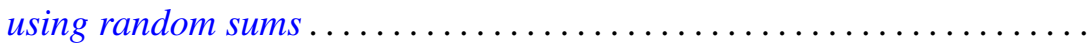

Karl-Theodor Eisele, Direct factorizations of measures .............. 79

Douglas Harris, Every space is a path component space ............. 95

John P. Holmes and Arthur Argyle Sagle, Analytic H-spaces, Campbell-Hausdorff formula, and alternative algebras.............

Richard Howard Hudson and Kenneth S. Williams, Some new residuacity criteria ..........................................

V. Karunakaran and Michael Robert Ziegler, The radius of starlikeness for a class of regular functions defined by an integral ....

Ka-Sing Lau, On the Banach spaces of functions with bounded upper

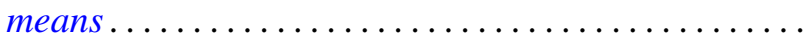

Daniel Paul Maki, On determining regular behavior from the recurrence formula for orthogonal polynomials................

Stephen Joseph McAdam, Asymptotic prime divisors and going down...

Douglas Edward Miller, Borel selectors for separated quotients ..

Kent Morrison, The scheme of finite-dimensional representations of an algebra

Donald P. Story, A characterization of the local Radon-Nikodým property by tensor products

Arne Stray, Two applications of the Schur-Nevanlinna algorithm ...

N. B. Tinberg, The Levi decomposition of a split $(B, N)$-pair ...

Charles Irvin Vinsonhaler and William Jennings Wickless, A theorem on quasi-pure-projective torsion free abelian groups of finite rank... 